\title{
A Mathematical Modeling of Infrared Neural Stimulation
}

Cesil S. Alex

University of South Florida

\author{
Advisors: \\ Arcadii Grinshpan, Mathematics and Statistics \\ Parveen Bazard, Global Center for Hearing and Speech Research \\ Robert Frisina, Global Center for Hearing and Speech Research \\ Problem Suggested By: Parveen Bazard
}

Follow this and additional works at: https://digitalcommons.usf.edu/ujmm

Part of the Mathematics Commons

UJMM is an open access journal, free to authors and readers, and relies on your support:

Donate Now

\section{Recommended Citation}

Alex, Cesil S. (2020) "A Mathematical Modeling of Infrared Neural Stimulation," Undergraduate Journal of Mathematical Modeling: One + Two: Vol. 10: Iss. 2, Article 1.

DOI: https://doi.org/10.5038/2326-3652.10.2.4914

Available at: https://digitalcommons.usf.edu/ujmm/vol10/iss2/1 


\title{
A Mathematical Modeling of Infrared Neural Stimulation
}

\begin{abstract}
Electrical stimulation is the gold standard for artificial neural stimulation. The greatest disadvantage with electrical stimulation is that it scatters in space and it is difficult to achieve specific point stimulation. Recently, infrared stimulation attracted attention to address this issue. Infrared stimulation works on the principle of heating the tissue, exploiting the energy of infrared lasers to heat the cellular aqueous solution that helps transfer the energy to the cell membrane without direct contact, and provides a discrete localization of stimulation as it does not spread in space like electric fields. In the present study, a heat transfer model for the temperature distribution was evaluated for infrared heating. All calculations were done for an aqueous medium, which can be a good initial representative of conditions in the human body, as it is comprised $60 \%$ of water. The Laplace transform was used to convert the convoluted function within the heat equation to a linear function. The variables were plotted to help identify and predict the most effective temperatures on the surface of neuron/cell that will be activated. This project describes the formulation of deriving temperature profiles used to predict optimal temperatures to activate neurons using advanced calculus tools.
\end{abstract}

\section{Keywords}

infrared stimulation, neurons, heat transfer, laplace transform, inverse laplace transform, complementary error function

\section{Creative Commons License}

(c) (i) (3)

This work is licensed under a Creative Commons Attribution-Noncommercial-Share Alike 4.0 License. 


\section{PROBLEM STATEMENT}

To find the effective temperature profiles at which infrared neurons/cells stimulation occurs using advanced calculus tools.

\section{MOTIVATION}

Current neurosensory implants and testing devices utilize electric currents to stimulate neurons/cells. As currents spread in space, it is not possible to achieve high spatial resolution for all stimulus situations $[3,7,13,16,17]$. In the last few decades, infrared stimulation garnered a great amount of attention to address this issue. There have been multiple reports of successful optical stimulation, specially, infrared stimulations with various pre-clinical in-vivo and in-vitro systems e.g., sciatic nerve, heart cells, SH-SY5Y neurons, DRG neurons and auditory systems, etc. $[1,2,4,9,10,12,19,21,23]$. To have a clearer understanding of the procedure as well as the concept which justifies the project, the following images of cochlear stimulation using infrared stimulation are provided. Figure 1 [14] shows an infrared device which is placed on to the basal turn of a cat cochlea. This device provides a heat source involving production of infrared waves. For this study, an optical fiber of $400 \mu \mathrm{m}$ in diameter was used to deliver the infrared pulses and would have been placed in the same area of the cochlea as Figure 1.

The second figure, Figure 2 [9], describes the effects of the infrared stimulation on the cochlea itself, and the activation of the cell in reference to the pathway of the infrared waves. The release of a protein labeled c-FOS has been correlated to the activation of neurons. This cFOS activation works congruently with the activation of the cochlear compound action potential. Cellular compound action potentials were assessed via c-FOS immunohistochemical 
staining. This measurement provided data related to the energy and time required to stimulate the neurons, as well as the directional effects of the laser on direct and indirect targets from the infrared pathway.

To better understand the optimal energy required for neural activation and resulting underlying cellular mechanisms, it is useful to predict heat temperature profiles during infrared stimulation. We used a one-dimensional transient heat conduction model in the present study [22]. For this heat transfer model, surrounding fluid is assumed stationary, as human body is made up of approximately $60 \%$ water and heat transfer is assumed to be by conduction only. A Laplace transform was used to solve the heat transfer equations. Utilizing the Laplace transform [8], we converted the time-space differential equation into a linear differential equation. The inverse Laplace transform provides the temperature distribution function, which graphed to find spatial and temporal temperature profiles during neural activation.

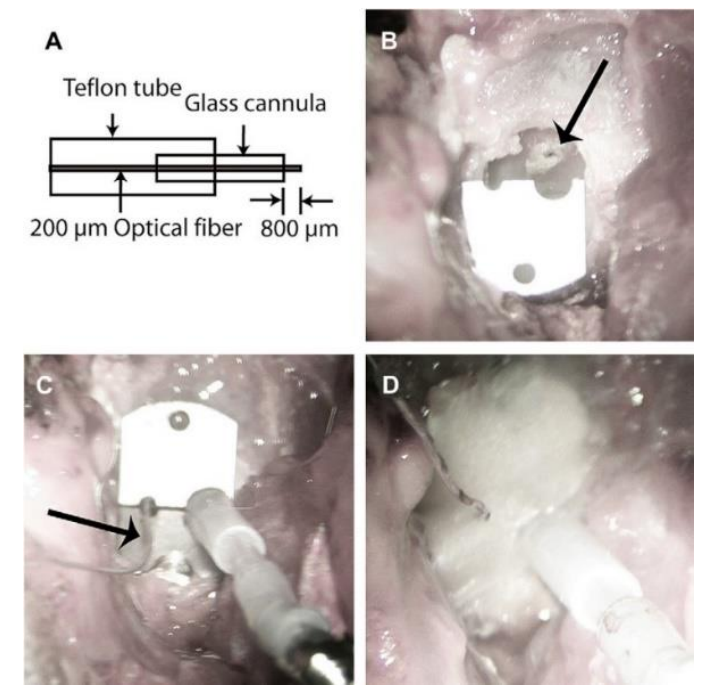

Figure 1: This image from [14] shows the placement of the optical device in cat cochlea. (A) Shows the length and placement of the optical fiber within the optical housing. (B) The arrow shows the drilled cochleostomy within the basal turn of the cochlea. (C) The arrow shows the round window recording electrode held by two stainless steel plates. (D) Shows how the assembly was held together by dental acrylic. 


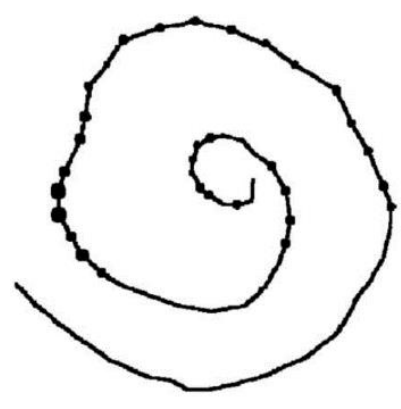

(a)



(b)

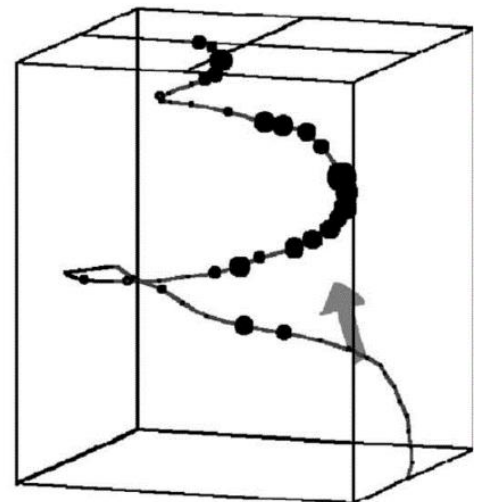

(c)

Figure 2: This three-dimensional representation of the affected cochlea, provided by [9], shows the effect of infrared stimulation. The spiraling line itself is a three-dimensional representation of the cochlea, and the arrow indicates the angle at which the laser was emitting the infrared waves. The circles represent the level of c-FOS activation within the cochlea. This activation is elicited by the infrared waves, which heat the water adjacent to the cell, which in turn activates the cell. As you may notice, the level of activation was specific, in direct correlation to the pathway of the laser.

\section{MATHEMATICAL DESCRIPTION AND SOLUTION APPROACH}

Firstly, we correlate the change in temperature as a function of the heat source in space and time with the following one dimensional (1-D) unsteady state heat conduction equation:

$$
\frac{\partial^{2} T}{\partial x^{2}}=\frac{1}{\alpha} \frac{\partial T(x, t)}{\partial t}
$$

This equation is used in conjunction with the following boundary conditions:

$$
T(x, 0)=T_{0} \text { and } T(x, t)=T_{0}
$$


At initial time $(t=0)$, the temperature at any point in space will equal its initial temperature $\left(\mathrm{T}_{\mathrm{o}}\right)$, and when the heat source is an infinite distance from the target, at any point in time, the temperature of the target will stay at its initial temperature $\left(\mathrm{T}_{\mathrm{o}}\right)$.

The heat flux of the heat source is dependent on the area to which the energy is distributed as well as the thermal conductivity of the medium [21], distance from the target, and time of conduction, and can be represented by the following equation:

$$
\frac{\partial T(0, t)}{\partial x}=\frac{q_{0}}{k}, \text { as } x \text { ranges from }(0, t) \text { for } \mathrm{t}>0
$$

Assuming the variable change of temperature as:

$$
T^{\sim}=T-T_{0}, \quad\left(\frac{1}{\alpha}\right) \frac{\partial T^{\sim}}{\partial t}=\frac{\partial^{2} T^{\sim}}{\partial x^{2}}
$$

The approach is subjected to the following conditions:

$$
T^{\sim}(x, 0)=0, \quad T^{\sim}(\infty, t)=0, \quad \frac{\partial T^{\sim}(0, t)}{\partial x}=\frac{q_{0}}{k}
$$

At time 0 , there will be no change in temperature of the target, with a similar result if the heat source is an infinite distance away from the target. At the interface of heat target (water) and source (laser), there is a flux continuity i.e., at distance $(x=0)$ away from the target, the heat flux of the target is equivalent to the heat flux of the heat source.

When taking the Laplace transform $T^{*}(x, s)=\int_{0}^{\infty} T^{\sim}(x, t) e^{-s t} d t$ one gets $[5, \mathrm{~V} .1 ; 11,15]$ :

$$
\left(\frac{1}{\alpha}\right)\left[s T^{*}(x, s)-T^{\sim}(x, 0)\right]=\frac{\partial^{2} T^{*}(x, s)}{\partial x^{2}}
$$




$$
\left(\frac{s}{\alpha}\right) T^{*}(x, s)=\frac{\partial^{2} T^{*}(x, s)}{\partial x^{2}}
$$

With the boundary conditions for the Laplace domain being:

$$
T^{*}(\infty, s)=0, \quad \frac{\partial T^{*}(0, s)}{\partial x}=\frac{q_{0}}{k s}
$$

When using (6) and (7) together, we obtain:

$$
T^{*}(x, s)=c_{1} e^{\sqrt{\frac{s}{\alpha}} x}+c_{2} e^{-\sqrt{\frac{s}{\alpha}} x}
$$

Using the boundary conditions within the transform, we understand that:

$$
c_{1}=0, \quad c_{2}=-\frac{q_{0} \sqrt{\alpha}}{k \sqrt{s^{3}}}
$$

and

$$
T^{*}(x, s)=-\frac{q_{0} \sqrt{\alpha}}{k \sqrt{s^{3}}} e^{-\sqrt{\frac{s}{\alpha}}}
$$

The inverse Laplace transform of function (8) [5, V.1, p.246] and (4) lead to equation (9). This is the one-dimensional heat conduction model [21] which portrays the effect of transient heat conduction in a semi-infinite medium exposed to constant heat flux:

$$
T-T_{0}=-\frac{q_{0}}{k}\left[2 \sqrt{\frac{\alpha t}{\pi}} e^{\frac{-x^{2}}{4 \alpha t}}-x \cdot \operatorname{erfc}\left(\frac{x}{2 \sqrt{\alpha t}}\right)\right]
$$

Function $\operatorname{erfc}(z)$ in (9) is the complementary error function which is derived from the error function $\operatorname{erf}$ (z) [5, V.1, p.387; 6]: 


$$
\operatorname{erfc}(z)=\frac{2}{\sqrt{\pi}} \int_{z}^{\infty} e^{-t^{2}} d t=1-\operatorname{erf}(z), \operatorname{erf}(z)=\frac{2}{\sqrt{\pi}} \int_{0}^{z} e^{-t^{2}} d t
$$

In equation (9), $q_{0}$ is identified as the heat flux of the infrared laser heat source, $k$ is the thermal conductivity of water, $\alpha$ represents the thermal diffusivity, $t$ represents time of heat conduction, and $x$ represents the distance of the target from the heat source $[21,18]$.

\section{Calculations}

In (9), $\alpha$ is the thermal diffusivity of water as the study [18] considers water as the medium in which heat transverses:

$$
\alpha=\frac{k}{\rho * C_{p}}=\frac{.608 \frac{\mathrm{W}}{\mathrm{m} \cdot{ }^{\circ} \mathrm{K}}}{1000 \frac{\mathrm{kg}}{\mathrm{m}^{3}} * 4180 \frac{\mathrm{J}}{\mathrm{kg}^{\circ} \mathrm{K}}}=1.455 * 10^{-7} \frac{\mathrm{m}^{2}}{\mathrm{~s}},
$$

where $k$ represents the thermal conductivity of water, $\rho$ represents the density of water, and $C_{p}$ represents the specific heat of water [20]; $q_{o}$, is representative of flux, and $\mathrm{W} / \mathrm{m}^{2}$ is calculated by the following $[21,8]$ : an area with a $400^{*} 10^{-6} \mathrm{~m}$ diameter with a laser pulse strength of $7.3 \mathrm{~mJ}$ for $10 \mathrm{~ms}$, is equivalent to $0.73 \mathrm{~W}$ [18]. The area of effect can be described as [18]: ((400/2) $\left.x 10^{-6}\right)^{2}$ $\pi=1.26^{*} 10^{-7} \mathrm{~m}^{2}$. To which, $q_{0}=0.73 / 1.26^{*} 10^{-7}=5.809 * 10^{6} \mathrm{~W} / \mathrm{m}^{2}$. The direction of $q_{0}$ is opposite of the direction of $x$ as the laser pulse direction is towards the target, and the movement of the source is away from the target. Thus $q_{0}=-5.809 * 10^{6} \mathrm{~W} / \mathrm{m}^{2}$, for any positive distance, $x$.

Once the representative variable values have been inputted to the equation, the following will result for a $0.73 \mathrm{mj} / \mathrm{ms}$ laser pulse: 


$$
\begin{gathered}
T-T_{0}=\frac{-\left(-5.809 \cdot 10^{6}\right) \frac{(J)}{\left(s \cdot m^{2}\right)}}{0.608 \frac{W}{m \cdot{ }^{\circ} K}} \\
\times\left[2 \sqrt{\frac{1.455 \cdot 10^{-7} \frac{W \cdot m^{2}}{J} t}{\pi}} \cdot \exp \left[\frac{-x^{2}}{4\left(1.455 \cdot 10^{-7} \frac{W \cdot m^{2}}{J}\right) t}\right]-\left[x \cdot \operatorname { e r f c } \left(\frac{x}{\left.\left.\left.2 \sqrt{1.455 \cdot 10^{-7} \frac{W \cdot m^{2}}{J} t}\right)\right]\right]}\right.\right.\right.
\end{gathered}
$$

The results will show the temperature change effect on a target from a heat source. The following temperature profile of the target was developed using the mathematical model with specific time and distance points. 


\section{RESULTS AND DISCUSSIONS}

\begin{tabular}{|c|c|c|c|c|c|c|c|c|c|}
\hline \multicolumn{10}{|c|}{$\begin{array}{c}\text { Temperature Profiles of Heat Conduction (change in temperature }\left({ }^{\circ} \mathrm{K}\right) \text { at } 0.73 \mathrm{~mJ} / \mathrm{ms} \\
\text { pulse) }\end{array}$} \\
\hline \multirow{2}{*}{$\begin{array}{l}\text { Distance } \\
(\mu \mathrm{m})\end{array}$} & \multicolumn{9}{|c|}{ Time $(\mu s)$} \\
\hline & $\mathbf{0}$ & 0.01 & 0.02 & 0.05 & 0.1 & 0.3 & 0.7 & 1.5 & 2.5 \\
\hline 0.00 & 0 & 0.41 & 0.58 & 0.92 & 1.30 & 2.25 & 3.44 & 5.04 & 6.50 \\
\hline 0.10 & 0 & 0.01 & 0.06 & 0.26 & 0.56 & 1.42 & 2.57 & 4.14 & 5.59 \\
\hline 0.15 & 0 & 0.00 & 0.01 & 0.12 & 0.34 & 1.10 & 2.20 & 3.73 & 5.17 \\
\hline 0.20 & 0 & 0.00 & 0.00 & 0.05 & 0.19 & 0.84 & 1.86 & 3.35 & 4.77 \\
\hline 0.30 & 0 & 0.00 & 0.00 & 0.00 & 0.05 & 0.46 & 1.31 & 2.68 & 4.03 \\
\hline 0.45 & 0 & 0.00 & 0.00 & 0.00 & 0.00 & 0.16 & 0.72 & 1.86 & 3.09 \\
\hline 0.70 & 0 & 0.00 & 0.00 & 0.00 & 0.00 & 0.02 & 0.22 & 0.94 & 1.89 \\
\hline 0.90 & 0 & 0.00 & 0.00 & 0.00 & 0.00 & 0.00 & 0.07 & 0.50 & 1.22 \\
\hline 1.20 & 0 & 0.00 & 0.00 & 0.00 & 0.00 & 0.00 & 0.01 & 0.17 & 0.59 \\
\hline 1.50 & 0 & 0.00 & 0.00 & 0.00 & 0.00 & 0.00 & 0.00 & 0.05 & 0.26 \\
\hline 2.00 & 0 & 0.00 & 0.00 & 0.00 & 0.00 & 0.00 & 0.00 & 0.00 & 0.05 \\
\hline
\end{tabular}

Table 1: The table shows how, as the distance increases from the heat source, the temperature change decreases, but as the heat source is held irradiating at a specific distance, the temperature of the target increases with time. This change in temperature is what is shown within the table between the dependent variables (distance) and independent variables (time).

\section{Temperature Profile of IR Stimulation} at a pulse of $0.73 \mathrm{~mJ} / \mathrm{ms}$

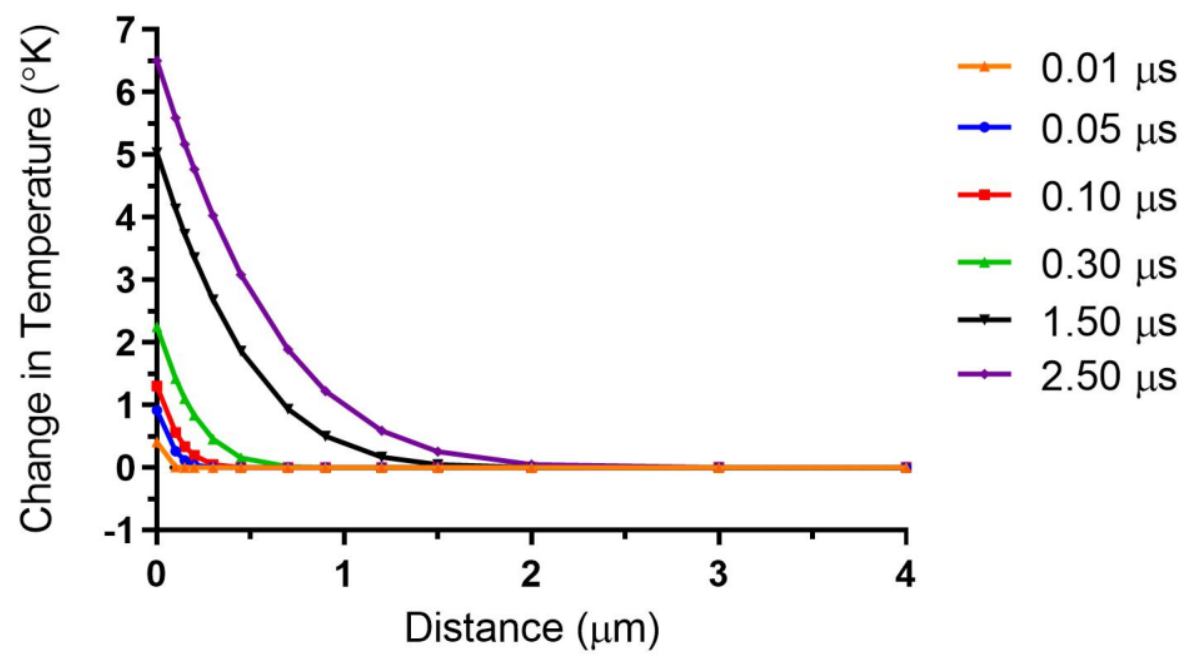


Graph 1: As expected, when the target is at a greater distance $(\mu \mathrm{m})$ from the heat source, the change temperature $\left({ }^{\circ} \mathrm{K}\right)$ decreases with respect to distance. When the heat source is activated for a greater amount of time, the change in temperature is larger. This is proven by the time stamps, showing the larger time points starting at a higher point in respect to the $y$-axis. It can be noticed that temperature decreases rapidly as distance increases. There is no temperature rise at distance far away from the source.

\section{Temperature Profile of IR Stimulation at a pulse of $3.7 \mathrm{~mJ} / \mathrm{ms}$}

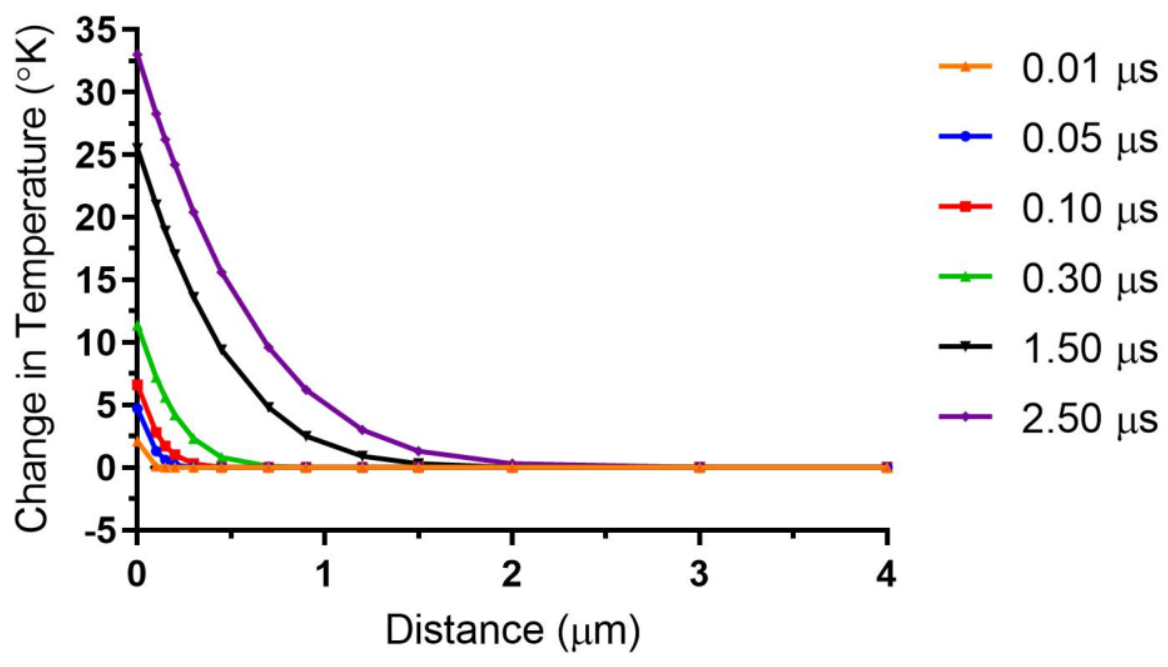

Graph 2: With the pulse strength of the laser increasing by $500 \%$, a similar increase in temperature change takes place. The pattern of the decrement remains the same as well to show that this model is most likely oversimplified to capture the reality of such an increase in a change in temperature. 


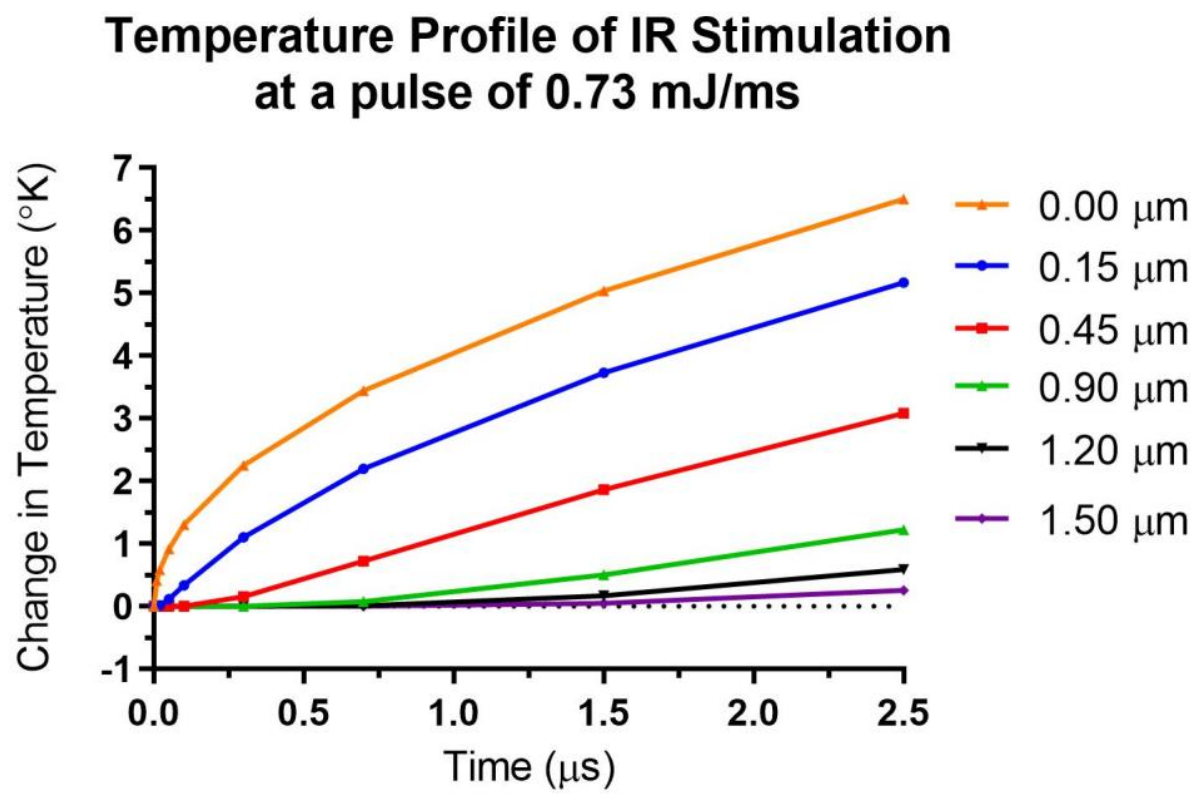

Graph 3: This graph shows the increase in change in temperature in reference to time, as the laser is held active on to the target. This slope of the increase becomes less steep as time of exposure increases. This is an expected pattern within the given range as a constant heat source will eventually plateau if the energy of the laser is allowed to dissipate within the given area of effect.

Temperature Profile of IR Stimulation at a pulse of $3.7 \mathrm{~mJ} / \mathrm{ms}$

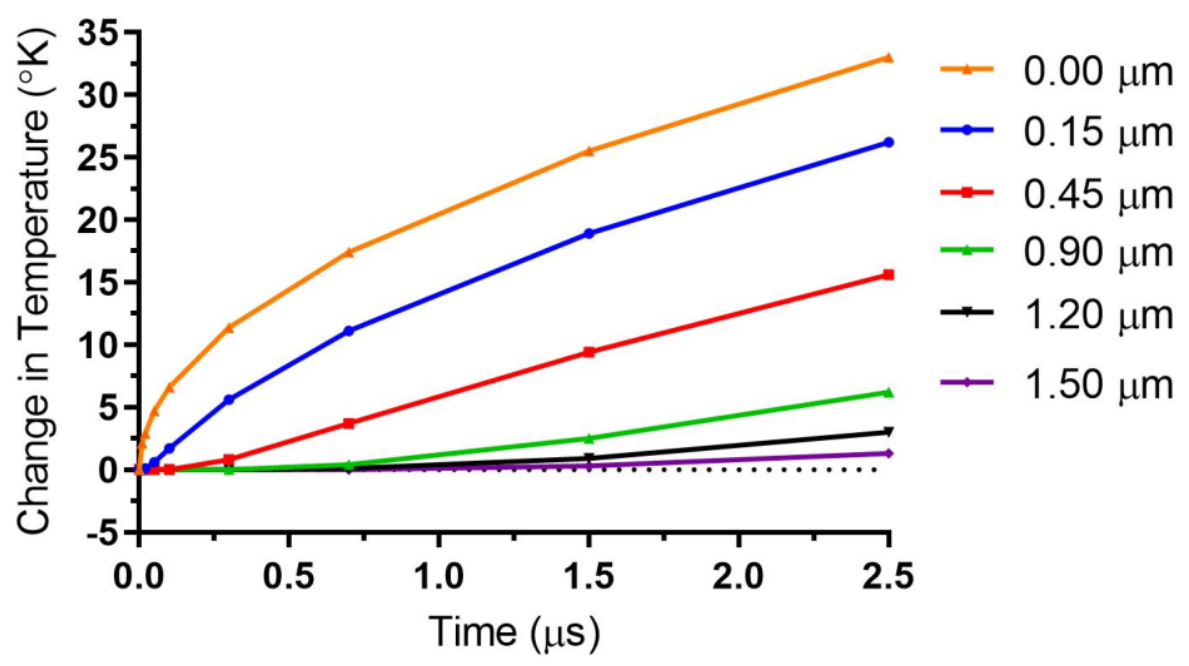

Graph 4: The change in pulse strength shows the increased change in temperature. The graph patterns again remaining like the lower strength laser pulse $(0.73 \mathrm{~mJ} / \mathrm{ms})$ when using similar distances to similar exposure time. 
Temperature profiles were calculated using a 1-D unsteady state heat transfer model for two separate pulse strengths, $0.73 \mathrm{~mJ} / \mathrm{ms}$ and $3.7 \mathrm{~mJ} / \mathrm{ms}$, reported by Shapiro et al [20]. Table 1 shows the change in temperature values at various times and distances with pulse strength of $0.73 \mathrm{~mJ} / \mathrm{ms}$. The same were plotted with time and distance variables. At a fixed time, temperature decreases very rapidly with distance as shown in Graph 1 and 2 for $0.73 \mathrm{~mJ} / \mathrm{ms}$ and $7.3 \mathrm{~mJ} / \mathrm{ms}$ strengths respectively. Similarly, at a fixed distance, temperature rises with time as shown in Graph 3 and 4 for two pulse strengths respectively.

In conclusion, an optimal temperature of neural stimulation can be derived by making use of the heat equation in congruence with the Laplace transform, as an avenue to relay the integration into a linear format. This format was then used to create a temperature profile to identify the change in temperature based on the distance of the heat source from the target, and the amount of time the heat source was being applied to the target.

Through the table and $\operatorname{graph}(\mathrm{s})$, it is shown that the greater the distance of the target from the heat source, the smaller the temperature change will be until the system temperature is established. If the heat source is continually being applied, the target shows to increase in temperature. With an optimal wavelength of applied energy, an efficient temperature would be reached to activate the cell, at a specific distance with a specific time of stimulation sustainment. This activation would occur by heating the cellular environment adjacent to the target cell, through the conduction of heat from the infrared heat source.

This paper presents a highly simplified 1-D unsteady state heat transfer model. It would be advised that further investigations utilize the information from the one-dimensional heat conduction modeling to develop a three-dimensional heat conduction model. Although one dimensional modeling was sufficient to show the effect of a heat source with a specific 
energy threshold to a target $x$ micrometers away, held at $s$ amount of time, in a linear fashion, heat is conducive in a three dimensional space involving $x, y$, and $z$ axis. Also, the cellular environment is assumed to be water, to which a more realistic situation would involve a saline environment. It would also be advised that a cell model like Hodgkin-Huxley model be used in conjunction to the heat transfer model to understand underlying cellular mechanism due to thermal pulses. This would not only help to estimate temperature thresholds for neural activation but also, provide appropriate safety limits in the heat transfer of the individual cells/cellular networks.

Lastly, for an effective in vivo laser-based neural or cardiac stimulation system, temperatures would have to be carefully controlled, so that over-heating, causing collateral tissue damage does not occur. Eventually, the laser system would have to be finely tuned and configured to deliver spatially precise energy, where the average changes in temperature at the cell membrane may be on the order of 1 degree or less.

\section{ACKNOWLEDGEMENT}

The work is supported by NIH grant - P01 AG009524. 


\section{NOMENCLATURE}

\begin{tabular}{|c|l|l|}
\hline Symbol & \multicolumn{1}{|c|}{ Description } & \multicolumn{1}{c|}{ Unit } \\
\hline$t$ & Time & Second \\
\hline$x$ & Distance of target from heat source & Meter \\
\hline$q_{o}$ & $\begin{array}{l}\text { Flux at } \mathrm{x}=0 \text {. i.e. the power of laser over } \\
\text { an area }\end{array}$ & $\mathrm{Joule} /\left(\mathrm{m}^{2} *^{\mathrm{s}} \mathrm{s}\right)$ \\
\hline$k$ & Conductivity of water is $0.608^{11}$ & $\mathrm{~W} /\left(\mathrm{m}^{*}{ }^{\circ} \mathrm{K}\right)$ \\
\hline$\rho($ rho $)$ & Density of water at $4{ }^{\circ} \mathrm{C}$ is $1000^{11}$ & $\mathrm{~kg} / \mathrm{m}^{3}$ \\
\hline$\propto($ alpha $)$ & Thermal diffusivity & $\mathrm{k} /\left(\rho^{*} \mathrm{Cp}\right)$ \\
\hline & $\begin{array}{l}\text { Specific heat of water at } 25^{\circ} \mathrm{C}, \text { is } \\
4180^{11}\end{array}$ & $\mathrm{~J} /\left(\mathrm{kg}{ }^{\circ} \mathrm{K}\right)$ \\
\hline
\end{tabular}




\section{REFERENCES}

1) Bazard, P., et al. (2017). "Nanoparticle-based Plasmonic Transduction for Modulation of Electrically Excitable Cells." Scientific Reports 7(1): 7803.

2) Carvalho-de-Souza, J. L., et al. (2015). "Photosensitivity of neurons enabled by cell-targeted ld nanoparticles." Neuron 86(1): 207-217.

3) Clark, G. (2004). Cochlear implants. Speech processing in the auditory system, Springer: 422-462.

4) Eom, K., et al. (2014). "Enhanced infrared neural stimulation using localized surface plasmon resonance of gold nanorods." Small 10(19): 3853-3857.

5) Erdélyi, A., Magnus, W., Oberhettinger, F., Tricomi, F.G. (1954). Tables of Integral Transforms. New York, McGraw-Hill.

6) Erdélyi, A., Magnus, W., Oberhettinger, F., Tricomi, F.G. (1981). Higher Transcendental Functions. Melbourne, Krieger.

7) Frigo, C., et al. (2000). "EMG signals detection and processing for on-line control of functional electrical stimulation." Journal of Electromyography Kinesiology 10(5): 351-360.

8) Gutierrez-Miravete, E. (2003). Exact Solution of Heat Conduction and Diffusion Problems. Mathematical Modeling of Heat Conduction and Mass Diffusion, Universidad Iberoamericana.

9) Izzo, A. I., Suh, E., Pathria, J., Walsh Jr., J.T., Whitlon, D.S., and Richter, C. (2007). "Selectivity of neural stimulation in the auditory system: a comparison of optic and electric stimuli." Journal of Biomedical Optics 12(2).

10) Izzo, A. I., Walsh Jr., J.T., Ralph, H., Webb, J., Bendett, M., Wells, J., and Richter, C. (2008). "Laser Stimulation of Auditory Neurons: Effect of Shorter Pulse Duration and Penetration Depth." Biophysical Journal (94): 3159-3166.

11) Levandosky, J. (2003) Laplace's Equation. Partial Differential Equations of Applied Mathematics

12) Li, W., et al. (2015). "Remote modulation of neural activities via near-infrared triggered release of biomolecules." Biomaterials 65: 76-85.

13) Littlefield, P. D., Vujanovic, I., Mundi, J., Matic, A.I., and Richter, C. (2010). "Laser Stimulation of Single Auditory Nerve Fibers." Laryngoscope(120): 2071-2082.

14) Moore, D. R. and R. V. Shannon (2009). "Beyond cochlear implants: awakening the deafened brain." Nature neuroscience 12(6): 686.

15) Muraoka, Y. (2002). "Development of an EMG recording device from stimulation electrodes for functional electrical stimulation." Frontiers of medical biological engineering: the international journal of the Japan Society of Medical Electronics Biological Engineering 11(4): 323-333.

16) Rajguru, S. M., Matic, A.I., Robinson, A.M., Fishman, A.J., Moreno, L.E., Bradley, A., Vujanovic, I., Breen, J., Wells, J.D., Bendett, M., and Richter, C. (2010). "Optical cochlear implants: Evaluation of surgical approach and laser parameters in cats." Hearing Research(269): 102-111. 
17) Richter, C., Bayon, R., Izzo, A.D., Otting, M., Suh, E., Goyal, S., Hotaling, J., and Walsh and J. T. Jr. (2008). "Optical stimulation of auditory neurons: Effects of acute and chronic deafening." Hearing Research(242): 42-51.

18) Rubinstein, J. T. and R. Hong (2003). "Signal coding in cochlear implants: exploiting stochastic effects of electrical stimulation." Annals of Otology, Rhinology Laryngology 112(9_suppl): 14-19.

19) Salo, R. W., et al. (2003). Apparatus and method for spatially and temporally distributing cardiac electrical stimulation, Google Patents.

20) Shapiro, M. G., Homma, K., Villarreal, S., Richter, C., and Bezanilla, F. (2012). "Infrared light excites cells by changing their electrical capacitance." Nature Communications(3): 736746.

21) Thompson, A. C., Stoddart, P.R., and Jansen, E.D. (2014). "Optical Stimulation of Neurons." Current Molecular Imaging(3): 162-177.

22) Thompson, W. J. (2000). Introduction to Transport Phenomena, Prentice Hall: 254-255.

23) Yoo, S., et al. (2014). "Photothermal inhibition of neural activity with near-infrared-sensitive nanotransducers." ACS Nano 8(8): 8040-8049. 